\title{
Development of a Computational Fluid Dynamics Model of the Left Atrium in Atrial Fibrillation on a Patient Specific Basis
}

\author{
Alessandro Masci ${ }^{1}$, Martino Alessandrini ${ }^{1}$, Luca Dedè ${ }^{2}$, Davide Forti ${ }^{2}$, Filippo Menghini ${ }^{2}$, Corrado \\ Tomasi $^{3}$, Alfio Quarteroni ${ }^{2}$, Cristiana Corsi ${ }^{1}$ \\ ${ }^{1}$ DEI, University of Bologna, Campus of Cesena, Bologna, Italy \\ ${ }^{2}$ CMCS, École Polytecnique Fédérale de Lausanne (EPFL), Lausanne, Switzerland \\ ${ }^{3}$ Deparment of Cardiology, Ospedale Santa Maria delle Croci, Ravenna, Italy
}

\begin{abstract}
Atrial Fibrillation is associated with a five-fold increase in the risk of cerebrovascular events, being responsible of 15-18\% of all strokes. The morphological and functional remodeling of the left atrium caused by atrial fibrillation favors blood stasis and, consequently, stroke risk. In this context, several clinical studies suggest that stroke risk stratification could be improved by using hemodynamic information on the left atrium and the left atrial appendage. The goal of this study was therefore to develop a patientspecific computational fluid dynamics model of the left atrium which may help quantify the hemodynamic implications of atrial fibrillation on a patient-specific basis. In this paper, we present the developed model as well as its application to one AF patient as a preliminary step forward towards an optimized stroke risk stratification and therapy delivery.
\end{abstract}

\section{Introduction}

Atrial Fibrillation (AF) is the most common form of arrhythmia worldwide. It has been estimated that the prevalence of AF in US is about 2.2 million including paroxysmal or persistent AF [1]. Moreover, recent studies [2] report that AF is expected to double in the next 50 years as the population mean age increases. Regarding the consequences of $\mathrm{AF}$, it is known that this pathology is an independent risk factor for stroke. It is associated with a four-to five-fold increased risk of cerebrovascular events [3], being responsible of $15-18 \%$ of all strokes. In this context, several clinical studies suggest that stroke risk stratification could be improved by studying the hemodynamic characteristics of the left atrium (LA) and mainly of the left atrial appendage (LAA) [4]. These observations are based on the consideration that $\mathrm{AF}$ alters the inter-atrial blood flow dynamics in such a way that favors blood stagnation in the LA and the LAA, thus implying an increase of the risk of thromboembolism.

Computational fluid dynamics (CFD) represents a unique tool to test different fluid dynamic conditions on a complex system, such as the cardiac blood flow, in a noninvasive fully controllable and reproducible way. Therefore, the aim of this work was the development of a patientspecific CFD model of the left atrium in AF to elucidate the hemodynamic implications of AF.

Despite the relevance of the problem, the literature on CFD modeling of the LA in AF is very scarce $[5,6]$. The most advanced effort in this sense is the paper by Koizumi et al. [6], where a realistic geometry segmented from a healthy volunteer was used as boundary condition. Yet, a healthy geometry does not account for anatomical remodeling effects which have a direct impact on LA hemodynamics in AF. Moreover, a simple motion model was used consisting of simple contraction/expansion in the direction from the mitral valve (MV) to the LA roof. Finally, fixed pressure values taken from the literature were used as boundary conditions and the MV was simulated as an on-off switch.

In our work we introduce several original contributions: patient specific LA anatomy and motion model are extracted directly from one AF patient by specifically designed image processing operations, moreover, the CFD model was constrained by assigning realistic inflow boundary conditions obtained from real Doppler measurements in $\mathrm{AF}$ patients.

\section{Methods}

In Figure 1, we show the workflow of the project, described in this Section.

\subsection{Patient data}

Clinical data were selected from one AF patient. In particular, CT data was acquired from a Philips Brilliance 64 CT scanner. Ten volumes (170 axial slices, pixel size 0.4 


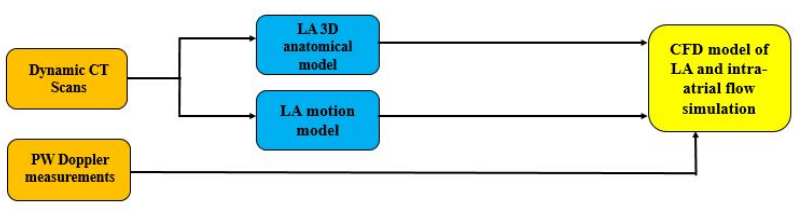

Figure 1. Project workflow.

$\mathrm{mm}$, slice thickness $1 \mathrm{~mm}$ ) spanning one cardiac cycle from the end of (ventricular) diastole were reconstructed by retrospective ECG gating. Moreover, in order to set realistic inflow boundary conditions for the CFD model, we used PW Doppler measurements at the mitral valve (MV) taken from the literature. For the LA, inflows and outflow are represented by the pulmonary veins (PVs) and the MV, respectively.

\subsection{Definition of the LA anatomical model}

The 3D LA anatomy, which includes, PVs and LAA was segmented from the first CT volume, through an image segmentation algorithm specifically designed.

First of all, we defined a region of interest (ROI) including the LA chamber. To perform LA segmentation, we segmented each slice of the CT volume and then reconstructed the 3D left atrial structure. For each slice, an initial segmentation was computed by intensity thresholding: the peak in the image histogram corresponding to the LA, called $I_{L A}$, was detected and used to define two thresholds, $\theta_{l o}$ and $\theta_{u p}$. They were defined as $\theta_{l o}=I_{L A}-q \cdot I_{L A}$ and $\theta_{u p}=I_{L A}+q \cdot I_{L A}$, where the constant $q$ is a percentage empirically defined: if $I_{L A}$ is $\geq 0.66, q$ is defined as $5 \%$; otherwise $q$ is set as $3 \%$. A set of morphological opening and closing operators were then applied to remove spurious regions. Finally, a curvature based level set was used to regularize the contours.

Moreover, in order to make the LA anatomical model compatible for a CFD simulation, we applied further processing, that consisted in a smoothing procedure (Laplacian smoothing) and in manual cutting of the inflow (i.e. the 4 PV's) and outflow (i.e. the MV) planes. Finally, a volumetric tetrahedral mesh was generated.

Figure 2 shows the result of the LA segmentation on four representative slices. Figure 3 illustrates the final LA mesh, which represented the computational domain for the fluid dynamics model.

\subsection{LA motion model}

The computation of the patient specific LA motion throughout the cardiac cycle was obtained by image registration of the full CT time sequence. Hereto, we computed the displacement $\mathbf{d}_{\mathbf{i} \rightarrow \mathbf{i}+\mathbf{1}}(\mathbf{x})$ between two successive CT
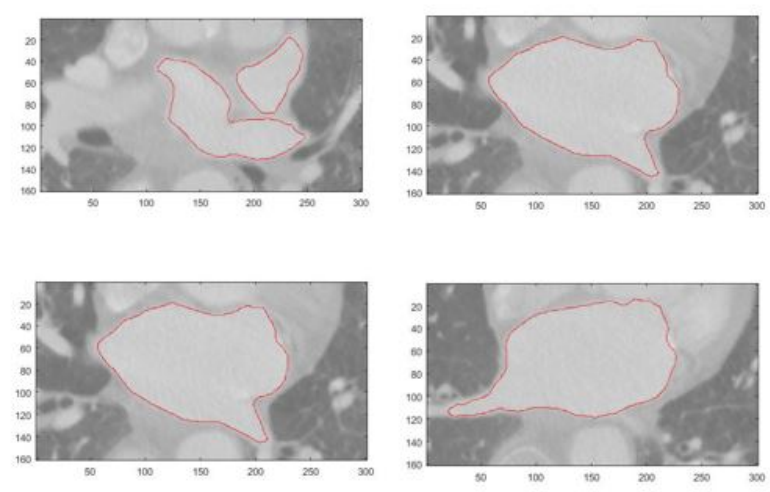

Figure 2. LA segmentation for the first patient in four slices of the CT volume.

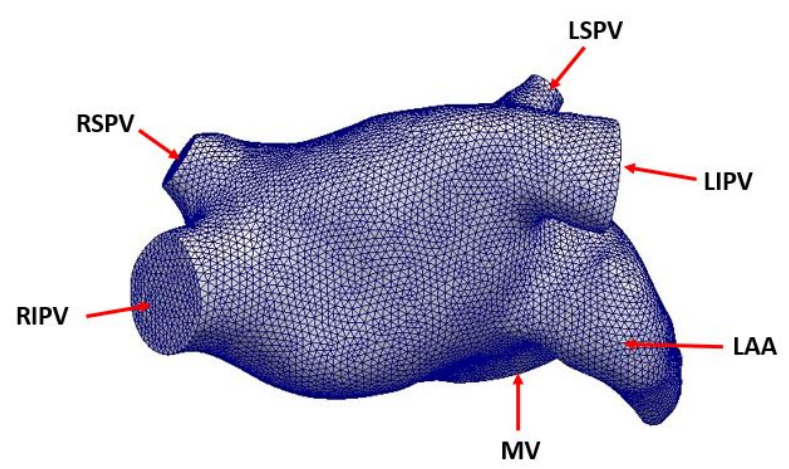

Figure 3. LA tetrahedral mesh. The most important LA anatomical structures are shown: right inferior PV (RIPV); right superior PV (RSPV); left inferior PV (LIPV); left superior PV (LSPV); LAA; MV

volumes $I_{i}(\mathbf{x})$ and $I_{i+1}(\mathbf{x})$ by using the mean squared difference as a similarity metric. The global displacement with respect to the first volume was computed by accumulating the successive inter-frame estimates by means of the recursive formula $d_{i \rightarrow 0}(x)=d_{i \rightarrow i-1}(x) \circ d_{i-1 \rightarrow 0}(x)$, with $\mathbf{d}_{\mathbf{0} \rightarrow \mathbf{0}}(\mathrm{x})=\mathbf{0}$. The displacement field obtained was then used to propagate the initial LA mesh, segmented at time 0 , over the ten CT frames of the cardiac cycle. Image registration and propagation of the initial mesh were performed in Elastix [7].

To increase temporal resolution for the stability of the CFD model, a continuous interpolation of the discrete mesh node positions was performed. Fourier series interpolation was used, which recovers a continuous and periodic function from the discrete data available, in agreement with the physiological periodicity of the heart motion. This way we were able to simulate an arbitrary number of cardiac cycles, which was necessary to remove unphysiological initial condition on fluid velocity. 


\subsection{CFD model}

The computational model consisted in a fluid governed by the incompressible Navier-Stokes equations written in the Arbitrary Lagrangian Eulerian (ALE) frame of reference [8], in order to take into account the motion of the computational domain. For the spatial discretization, we considered the Finite Element (FE) approximation, using scalar Lagrangian basis functions of polynomial degree $r \geq 1$, with a suitable stabilization scheme [9]. Regarding the temporal discretization, a second order semi-implicit backward differentiation formula scheme was used [9]. Furthermore, we defined the boundary conditions at the four pulmonary veins and at the mitral valve. First at all, we imposed a parabolic velocity profile at each PV. About the choice of the flowrate to assign at each PV, we considered a representative mitral valve flowrate $Q_{O}$ from [6]. We carried out this choice because, for the patient analyzed in this study, the PW Doppler velocity measurements at the MV were not available. In this way, the flowrate at each PV was then obtained by mass balance: $Q_{1}^{p v}+Q_{2}^{p v}+Q_{3}^{p v}+Q_{4}^{p v}+Q^{O}+Q_{\text {wall }}=0$, where $Q_{i}^{p v}, i=\{1,2,3,4\}$ are the flowrates at each PV and $Q^{O}$ is the desired MV flow rate. $Q_{\text {wall }}$ is the flux associated to the LA volume variation, i.e. $Q_{\text {wall }}=d V / d t$, being $V$ the instantaneous LA volume. By defining $Q_{t o t}^{p v}=$ $Q_{1}^{p v}+Q_{2}^{p v}+Q_{3}^{p v}+Q_{4}^{p v}$, the flowrates at each PV were then computed as follows: $Q_{l}^{p v}=\left(A_{l} / A_{t}\right) Q_{t o t}^{p v}-Q_{l}^{w}$, where $A_{l}$ represents the sectional area and $A_{t}$ is the sum of all PVs sectional areas. $Q_{l}^{w}$ represents the flow due to the mesh velocity for each PV considering that the PVs sections move and their area changes during the cardiac cycle. Regarding the outflow, i.e. MV, we imposed a natural boundary conditions to penalize backflow. The CFD simulations were performed through the LifeV library [10].

\subsection{CFD simulation}

We simulated two conditions: one corresponding to the sinus rhythm (SR) and one corresponding to AF condition. SR and AF differ in the motion model employed: SR employs the patient specific motion model extracted from the CT volumes described previously, whereas AF was instead simulated by applying, independently to each node, a random displacement, that was kept small $(\approx 0.01 \mathrm{~mm})$ to avoid numerical issues arising from an excessive worsening of the mesh quality. Moreover, in AF, we changed the inflow boundary by removing the A-wave to account for the lack of atrial contraction. Three cardiac cycles were simulated and the results on the third simulated cycle is reported in the following sections.

\section{Results}

In Figure 4(a) we show the blood velocity within the LA. During LV systole MV velocity is zero, as expected. However, we noticed a velocity increase up to $10 \mathrm{~cm} / \mathrm{s}$ in proximity of the four PVs in SR due to atrial diastole. We did not see this velocity increment at the four PVs in AF simulation, due to the random motion model. At the beginning of LV diastole (first row), the MV velocity reached a peak value of about $55 \mathrm{~cm} / \mathrm{s}$ (E-wave) in both the simulated conditions. Moreover, we noticed an expected increase of the PV velocity (mean value $40 \mathrm{~cm} / \mathrm{s}$ ). During atrial systole (second row) the peak velocity of the A-wave was between 35 and $40 \mathrm{~cm} / \mathrm{s}$, while the A-wave was missing in AF. Moreover, the differences in the complexity of the velocity distribution within the LA in the two conditions is appreciable in Figure 4(a).

Additional flow features, including small vortexes, kinetic energy and enstrophy, were computed, but are not discussed here due to the lack of space.

Flow dynamics in the LAA were specifically analyzed given its importance for clot formation [4]. Figure 4(b) shows flow velocity inside the LAA during atrial contraction for SR and AF simulations (first and second row, respectively). Note the reduced velocity in AF which can imply a reduced blood washout. To quantify blood washout we performed a specific study. We populated the LAA with 500 fluid particles at the beginning of the simulation and we counted how many particles remained inside the LAA after each cardiac cycle. We considered the first three cardiac cycles for both the conditions. After 3 cycles, 26\% of the particles remained in the LAA in SR, while $45.6 \%$ remained in AF (Table 1). Similar results were obtained in the second patient. Therefore, AF promotes an expected reduced washout of the LAA implying blood stasis, which in the long term might be indicative of the generation of blood clots.

\begin{tabular}{|c|c|c|}
\hline Cardiac cycle & SR condition & AF condition \\
\hline 0 & 500 & 500 \\
\hline 1 & 500 & 500 \\
\hline 2 & 478 & 480 \\
\hline 3 & $130(26 \%)$ & $228(45 \%)$ \\
\hline
\end{tabular}

Table 1. Number of particles which remained in the LAA at the end of each cardiac cycle in SR and AF for the first patient. Cardiac cycle $=0$ indicate the beginning of the simulation.

\section{Conclusions}

We presented our initial efforts towards the development of a patient specific CFD model of AF. To our knowledge, 


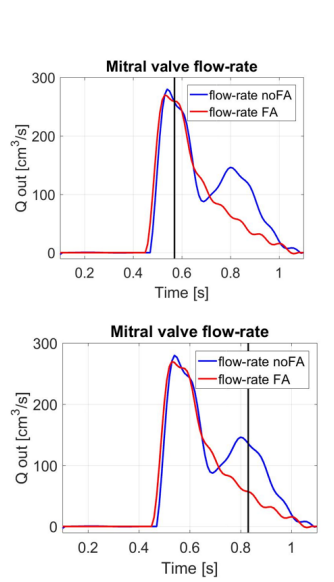

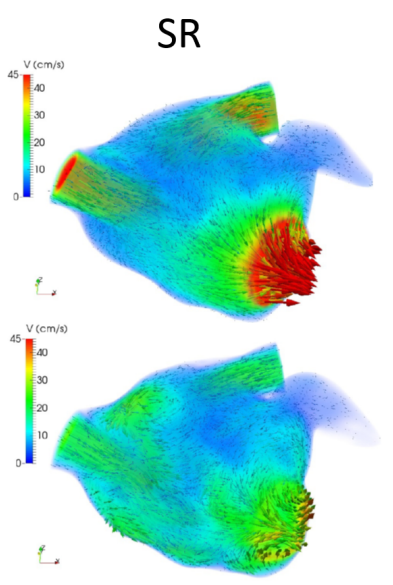

(a)

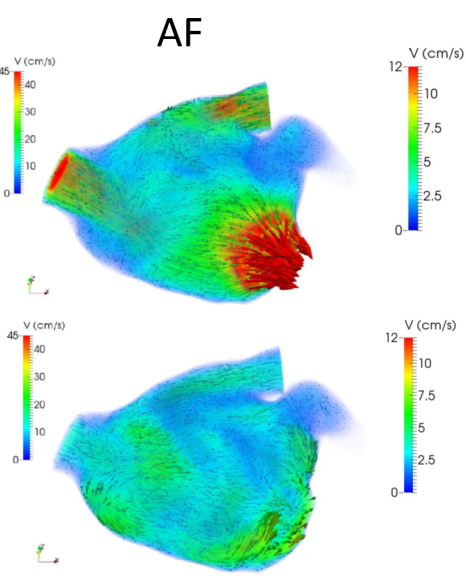

SR

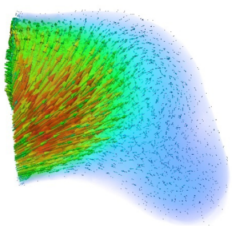

$\mathrm{AF}$

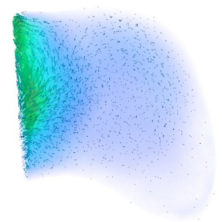

(b)

Figure 4. (a) Computed LA blood flow. First column reports the computed flow rate at the MV in SR (blue) and AF (red). Each row correspond to a different time in the cardiac cycle, denoted by the vertical black marker: LV filling (E-wave); atrial systole (A-wave). The velocity distributions within the LA are reported in the second (AF) and third (SR) column. (b) Blood flow in the LAA during atrial contraction.

this is the first presented in literature. The model returned realistic blood flow patterns both at SR and during AF. In addition, it confirmed that AF episodes result in a reduced washout of the LAA which might lead to thrombi formation. Our future work includes bench-marking the model against experimental flow measurements as with phase contrast MR or particle velocimetry imaging. The dataset will be increased by including control cases and more $\mathrm{AF}$ patients including a diverse range or morphology and contraction patterns. With our model we aim to enable optimized patient stroke risk assessment and therapy delivery in AF patients.

\section{References}

[1] Chugh SS, Blackshear JL, Shen WK, Hammill SC, Gersh BJ. Epidemiology and natural history of atrial fibrillation: clinical implications. Journal of the American College of Cardiology 2001;37(2):371-378.

[2] Camm AJ, Kirchhof P, Lip GY, Schotten U, Savelieva I, Ernst S, et al. Guidelines for the management of atrial fibrillation. European Heart Journal 2010;31(19):2369-2429. ISSN 0195-668X.

[3] Wolf PA, Abbott RD, Kannel WB. Atrial fibrillation as an independent risk factor for stroke: the framingham study. Stroke 1991;22(8):983-988.

[4] Gupta DK, Shah AM, Giugliano RP, Ruff CT, et al. Left atrial structure and function in atrial fibrillation: Engage aftimi 48. European Heart Journal 2014;35(22):1457-1465. ISSN 0195-668X.
[5] Zhang LT, Gay M. Characterizing left atrial appendage functions in sinus rhythm and atrial fibrillation using computational models. Journal of Biomechanics 2008; 41(11):2515-2523. ISSN 00219290.

[6] Koizumi R, Funamoto K, Hayase T, Kanke Y, Shibata M, Shiraishi Y, Yambe T. Numerical analysis of hemodynamic changes in the left atrium due to atrial fibrillation. Journal of biomechanics 2015;48(3):472-478.

[7] Klein S, Staring M, Murphy K, Viergever MA, Pluim JPW. elastix: A toolbox for intensity-based medical image registration. IEEE Transactions on Medical Imaging Jan 2010; 29(1):196-205. ISSN 0278-0062.

[8] Reymond P, Crosetto P, Deparis S, Quarteroni A, Stergiopulos N. Physiological simulation of blood flow in the aorta: comparison of hemodynamic indices as predicted by 3-d fsi, 3-d rigid wall and 1-d models. Medical Engineering Physics 2013;35(6):784-791.

[9] Forti D, Dedè L. Semi-implicit BDF time discretization of the Navier-Stokes equations with VMS-LES modeling in a high performance computing framework. Computers Fluids 2015;117:168-182.

[10] Malossi C, Deparis S. Lifev development guidelines. The LifeV Project 2009;.

Address for correspondence:

Cristiana Corsi, $\mathrm{PhD}$

DEI, University of Bologna

Via Risorgimento 2, 40136 Bologna, Italy

E-mail address: cristiana.corsi3@unibo.it 